\title{
Foul 2050: Thoughts on Physical Interaction in Human-Robot Soccer
}

\author{
Sami Haddadin, Tim Laue, Udo Frese and Gerd Hirzinger
}

\begin{abstract}
The RoboCup community has one definite goal: winning against the human world soccer champion team by the year 2050 [1]. This implies real tackles and fouls between humans and robots and rises safety concerns for the robots and even more important for the human players. Nowadays, similar questions are discussed in the field of physical humanrobot interaction (pHRI), but mainly in the context of industrial and service robotics applications.

Our paper is an attempt for a pHRI view on human-robot soccer. Technically, this will be years ahead, but still we believe it is enlighting to analyze the RoboCup vision from the perspective of pHRI. We take scenes from the 2006 FIFA World Cup as examples and discuss what could have happened if one of the teams consisted of robots instead of humans.
\end{abstract}

\section{INTRODUCTION}

Soon after establishing the RoboCup competition in 1997, the RoboCup Federation proclaimed an ambitious long term goal.

"By mid-21st century, a team of fully autonomous humanoid robot soccer players shall win the soccer game, comply with the official rule of the FIFA, against the winner of the most recent World Cup."

\section{H. Kitano and M. Asada [1]}

Soccer is a contact sport and injuries of players are frequent [2]. Even more, the FIFA rules state explicitly, that

"Football is a competitive sport and physical contact between players is a normal and acceptable part of the game. [...]"

Laws of the game, 2006 [3]

For a soccer match between humans and robots this implies physical human-robot interaction (pHRI) including tackles and fouls between humans and robots. In order to come closer to that vision, an evaluation of the fundamental requirements and challenges the human presence would bring into such a match is, in our opinion, absolutely crucial and definitely still an open issue. This makes not only sense with respect to e.g. ensuring human safety but as well to define requirements a robot has to fulfil in order to be able to withstand the enormous strains posed by such a real soccer game. These problems can only be approached and tackled if one sees the robotic and biomechanical aspects as complementary.

S. Haddadin and G. Hirzinger are with Institute of Robotics and Mechatronics, DLR - German Aerospace Center, Wessling, Germany \{sami.haddadin, gerd.hirzinger\}edlr.de

T. Laue is with FB 3 - Mathematics and Informatics, University of Bremen, Germany timlauedinformatik. uni-bremen. de

U. Frese is with Safe and Secure Cognitive Systems, DFKI, Germany Udo.Frese@dfki.de
The contribution of this paper is to shed light on the pHRI aspects of such a hypothetical human-robot match. Therefore, we use two matches from the recent (2006) FIFA World Cup in Germany as examples and analyze them with respect to scenes with physical interaction (cf. Tab.I). We relate these interactions to recent results in pHRI and sports science by imagining what would have happened if one of the opponents was a robot.

While winning against the human world champion is the official RoboCup grand challenge, RoboCup research and literature is mostly focussed on incremental progress from tournament to tournament. We believe, in order to take a vision seriously, it is essential to once in a while step back and analyze the challenges involved without focussing on short term ideas for solutions. This has been the motivation for our paper. We try to point out solutions where available but our main contribution is to discuss what requirements a human-robot soccer match would imply. We hope this also helps to guide incremental technical research into a direction that is fruitful in the long run.

In the domains of industrial and service robotics, robots are designed to cause absolutely no harm to any human. Presumably, such a robot could never win. However, we demand that a human-robot match should not be more dangerous than an ordinary soccer match. Hence, we focus on situations, where a robot is expected to potentially cause more injury than a human player.

Additionally, we contribute some mechanical considerations on how the robot can endure such interactions and meet the performance needed for competing with humans. In particular, we compare joint torque and velocity data from human athletes [4] with a state of the art robot arm [5] ${ }^{1}$ and discuss requirements for physical interaction control.

The paper is organized as follows: Section II reviews the current state of the art in pHRI; Section III classifies and discusses selected world cup scenes; and Section IV presents the mechanical considerations mentioned above.

\section{State of the ART In Physical Human-Robot INTERACTION}

Recently, there is increasing interest in domestic and industrial service robots that allow physical interaction [6], [7], [8]. The goal of robots and humans coexisting in the same physical domain poses various fundamental problems for the entire robotic design. Unlike classical ones, these robots take into account for the hardware, control and planning that

\footnotetext{
${ }^{1}$ This arm has similar inertial properties to the human leg and is faster than a typical humanoid robot's leg.
} 
a)

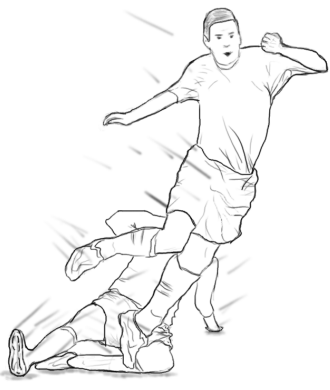

b)

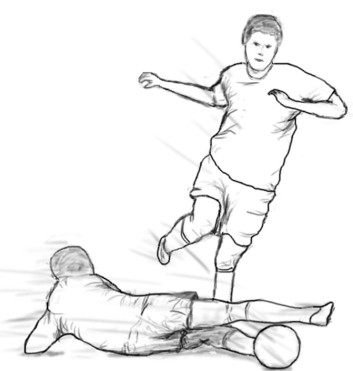

c)

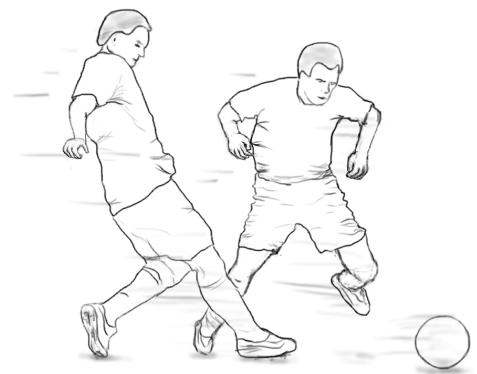

Fig. 1. Typical tripping scenes: a, b) A player slides to the ball and touches his opponent's legs (T2,T1). c) A player trips his opponent up (T3).

the environment is partially unknown. In order to allow a robot or even a human-like robotic system to physically interact with its environment, it is usually equipped with proprioceptive sensors, such as Cartesian force-/torque and joint torque sensors [5] and/or tactile ones such as sensitive skins (especially for hands [9]). Alternatively, backdriveable motors are used to passively react to external forces [10]. Walking humanoid robots such as HRP-2 [11] are usually equipped with additional acceleration sensors and gyroscopes which provide inertial information about body motion, support walking and in particular help to counterbalance unexpected interactions.

The most widely used approach to physically interact is probably impedance control and its related schemes, introduced in the pioneering work of Neville Hogan in [12] and extended to many classes of robots. This type of controller imposes a desired physical behavior on the robot, such as e.g. a second order mass-spring-damper system. Consequently, it allows to realize compliance of the robot by means of control.

Interaction with an impedance controlled robot is very robust and intuitive, since in addition to the commanded trajectory, a disturbance response is defined. A major advantage of impedance control (with impedance causality) is that discontinuities like contact-non-contact do not create such stability problems as for example hybrid force control [13]. However, many open questions still have to be tackled from a control point of view, such as how to adjust the impedance according to the current state.

Apart from such control issues, mechanical design plays a fundamental role in bringing humans and robots spatially closer. Classical robots are usually characterized by a stiff coupling between motor and link inertia. Therefore, in case of an impact the motor inertia cannot be neglected. However, for flexible-joint robots this does not fully apply for very hard impacts [14]. An interesting and promising paradigm currently rearising in robotics design is antagonism [15], [16], or more generally variable stiffness/impedance actuation. The idea is to implement joint compliance not by means of control but via intrinsically compliant joints, inspired by the unquestionably successful design of human and animal muscles. This intrinsic compliance can be adjusted according to the task and generally reduces joint torques of the (flexible-joint) robot (in flexible-joint robots, one has to distinguish between motor and joint torques). A direct implication of such joint stiffness reduction is a decoupling effect between motor and link inertia at high frequencies, growing with decreasing joint stiffness. As will be outlined later, introducing passive compliance into the mechanical robot design is crucial to cope with the enormous loads and velocities occurring during typical instep soccer kicks.

As Asimov already noted very early, safety has priority if robots are close to humans [17]. Fundamental work on human-robot impacts under worst-case conditions and resulting injuries was carried out in [14], [18], [19], taking a look at moderate robot speed up to $2 \mathrm{~m} / \mathrm{s}$. During such unexpected collisions, various injury sources exist: fast impacts, clamping, slow quasistatic loading, or sharp tools. Current results indicate that a robot, even with arbitrary mass driving not much faster than $2 \mathrm{~m} / \mathrm{s}$ is not able to become dangerous to a non-clamped human with respect to typical severity measures used in automobile industry [14]. This does not rule out other injuries, such as fractures, cuts, or laceration, but it indicates that typical physical human-robot interaction is much less dangerous than indicated in earlier work.

\section{Physical Interaction In SOCCER}

In this section, we separate possible physical interactions occurring in soccer into different classes and discuss their injury potential for the human and the robot. A set of scenes from the recent FIFA world championship serve as examples. We chose the final (Italy vs. France) as well as one of the toughest ${ }^{2}$ matches of the tournament (Portugal vs. the Netherlands) for our analysis. Tab.I shows the analyzed and classified scenes and which players were involved. To investigate possible injury mechanisms, frequently involved body parts need to be spotted. According to [20], adult soccer injury spreads almost over the whole body, but especially concerns the limbs (arm 15\%, hand 9\%, ankle 32\%, and knee $26 \%$ ), the back (5\%), and the head (11\%), whereas the rest of the torso seems to be in less danger. Injury causes were analyzed in [21], indicating that collisions with opponents $(22.4 \%)$ or the ball $(20.3 \%)$, incidents while being in motion $(17.1 \%)$ or after falling down $(8.2 \%)$ are most frequent. In this paper, we focus on these dominant injury sources and mechanisms.

\footnotetext{
${ }^{2} 16$ yellow cards (including four second cautions) denoted the maximum value of the whole tournament.
} 


\begin{tabular}{|c|c|c|c|}
\hline Scene & Time & Description & Figure \\
\hline $\mathrm{T} 1$ & $30: 37$ & Costinha skids to Cocu's feet and overthrows him (yellow card). & $1 b$ \\
\hline $\mathrm{T} 2$ & $72: 03$ & Heitinga runs fast while his leg is thrusted by sliding Deco (yellow card). & $1 \mathrm{a}$ \\
\hline $\mathrm{T} 3$ & $86: 33$ & Ooijer trips Petit up. & $1 \mathrm{c}$ \\
\hline $\mathrm{T} 4$ & $94: 52$ & Van Bronckhorst trips Tiago up (yellow card). & \\
\hline I1 & $92: 50$ & Ricado and Kuyt both jump to reach a ball, colliding in the air. & \\
\hline L1 & $06: 52$ & Bouhlarouz hits Ronaldo's thigh with his boot while his leg is half-elongated (yellow card). & \\
\hline L2 & $38: 20$ & Costinha tries to play the ball but hits Ooijer's shin. & \\
\hline L3 & $41: 50$ & $\begin{array}{l}\text { Robben and Valente both approach a high ball, Valente jumps with elongated leg and hits } \\
\text { Robben's chest. }\end{array}$ & $3 a$ \\
\hline L4 & $62: 00$ & $\begin{array}{l}\text { Bouhlarouz approaches Figo from the side to gain access to the ball. While running, he hits } \\
\text { Figo with his elbow in the face. }\end{array}$ & $3 \mathrm{c}$ \\
\hline L5 & $79: 40$ & $\begin{array}{l}\text { Kuijt skids towards Ricardo and hits the goalkeeper's shank with his boots causing a minor } \\
\text { injury. }\end{array}$ & $4 \mathrm{a}$ \\
\hline L6 & $87: 38$ & Simão steps on the goalkeeper van der Saar. & \\
\hline $\mathrm{P} 1$ & $14: 48$ & Kuyt and Carvalho run leaning against each other in parallel, Kuyt falls. & \\
\hline $\mathrm{P} 2$ & $61: 50$ & Van der Vaart and Figo chase the ball and push against each other. & $4 \mathrm{~b}$ \\
\hline T5 & $04: 22$ & Zambrotta hits Vieira's supporting leg and Vieira falls badly (yellow card) & \\
\hline $\mathrm{I} 2$ & $00: 35$ & Cannavaro and Henry collide with their trunks, Henry falls. & $2 \mathrm{a}$ \\
\hline $\mathrm{I} 3$ & $34: 03$ & Materazzi moves forward, Ribery backward, both collide. & \\
\hline I4 & $65: 23$ & Ribery and Grosso jump, Grosso lands on Ribery's back. & \\
\hline I5 & $71: 39$ & Camoranesi is running and gets blocked by standing Abidal, Camoranesi falls. & \\
\hline I6 & 79:04 & Cannavaro and Zidane jump. Cannavaro jumps higher and drags Zidane to the ground. & \\
\hline I7 & E01:13 & Makelele and Gattuso jump. Makelele jumps higher and lands on Gattuso's back. & $2 b$ \\
\hline I8 & E04:16 & Gattuso rushes into Malouda while approaching the ball. & \\
\hline L7 & $10: 47$ & Sagnol runs into Grosso, their knees collide (yellow card). & $3 b$ \\
\hline L8 & $31: 53$ & Ribery steps on Zambrotta's ankle joint. & \\
\hline L9 & $72: 03$ & Toni kicks the ball in a $180^{\circ}$ rotation and hits Thuram's knee with his shin. & \\
\hline L10 & E04:16 & Malouda hits Gattuso's face with his lower arm. & \\
\hline P3 & $35: 07$ & Thuram and Toni run with entangled arms. & \\
\hline $\mathrm{P} 4$ & $45: 10$ & Zidane pushes Gattuso. & \\
\hline P5 & $74: 57$ & Malouda jumps to head the ball and gets pushed by Zambrotta. & \\
\hline P6 & E02:17 & Malouda and Cannavaro run parallel and push each other. In the end, both fall. & \\
\hline
\end{tabular}

TABLE I

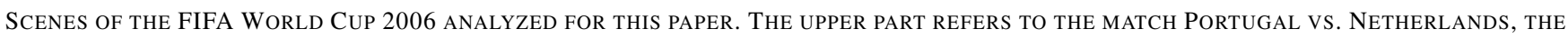
LOWER TO ITALY VS. FRANCE (FINAL).

\section{A. Tripping and Getting Tripped up (T)}

Tripping at high speed over the opponent's legs seems to have a reasonably high injury potential and is a commonly observed action. It is not necessarily an intended foul, but can be a legal tackling which aims at the ball. We could roughly divide three categories of tripping someone up in soccer:

- Hitting the opponent's feet intentionally by a sliding tackle (Fig. 1a, b ${ }^{3} ; \mathrm{T} 1, \mathrm{~T} 2$ ).

- Hitting the opponent's feet or legs unintentionally while chasing the ball (Fig. 1c; T3, T4).

\footnotetext{
${ }^{3}$ To avoid any copyright conflicts, we sketched the most significant situations.
}

- Directly attacking the opponent's legs (T5) without any chance of playing the ball.

This kind of interaction usually causes two mechanisms of injury: fractures of lower and upper extremities, ankle or knee injuries by direct contact [20], and indirect ones from resulting tumble. Soft covering of the robotic leg can decrease this injury potential dramatically and protects the robot's structure. Because tripping can be a sudden situation with little time to actively react, an overall compliant covering of the robot seems to be required. This is because the robot could fall in a more or less arbitrary direction with an undefined impacting zone. Passive compliance in the joints seems to be an effective countermeasure to intrinsically decouple impacting masses and decrease potential danger. 
a)

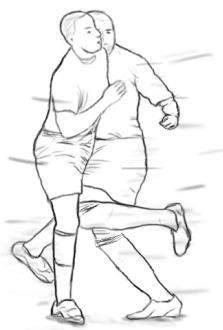

b)

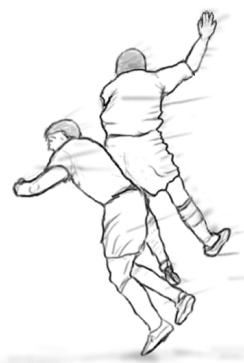

Fig. 2. Trunk impacts: a) Two players run into each other (I2). b) During a header, two players collide. Afterwards, one of them falls upon his opponent (I7).

Apart from the properties mentioned in Sec. II it makes the entire structure more flexible. The humanoid robot HRP-2 is e.g. already able to fall down and stand up (without gaining momentum).

An absolutely necessary action the robot has to perform is minimizing impact forces on its body similar to humans by preshaping its limbs.

\section{B. Trunk and Head Impacts (I)}

Trunk and head impacts occur frequently and they are usually caused by

- Two players colliding while running towards each other (Fig. 2a; I2, I3, I8)

- One player body-checking the other player (I5, I8)

- Two players jumping back to back at each other when fighting for a header (Fig. 2b; I1, I6, I7)

- One player falling on the other one who is lying on the ground (I4, I7)

This particularly limits the robot's weight because kinetic energy is according to [22], [23] a (limited) indicator of head injury and is (not directly) related to chest injury. Therefore, the robot's weight has to be similar to the one of professional soccer players. This was also demanded by Burkhard et al.: "The robots should have heights and weights comparable to the human ones (at least for safety reasons) [...]" [24]. According to [25], the average weight of the FIFA Worldcup 2002 participants was $75.91 \pm 6.38 \mathrm{~kg}$. For much higher robot masses, the situation of a human clamped on the ground by a robot that outweights him, poses significant danger to the limbs, chest, and other body parts. The weight of current humanoids, such as ASIMO (54kg), HRP-2/3 (58/65kg), WABIAN $(64.5 \mathrm{~kg})$ or HUBO $(57 \mathrm{~kg})$ is generally less than the ones of an average soccer player but all of them are fairly smaller.

Apart from limiting the robot weight, its body surface should definitely be padded to avoid human injuries from sharp edges, resulting in fractions, lacerations or cuts which already occur at blunt impacts [20]. Very sensitive parts of the human body are the spinal column and facial bones, having relatively low fracture forces [26] and necessitating compliant behavior of the robot's back (Fig. 2b). Nevertheless, one should keep in mind that headers require a hard contact a)

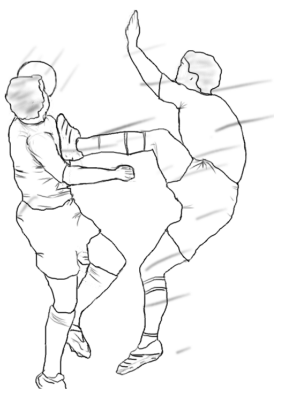

b)

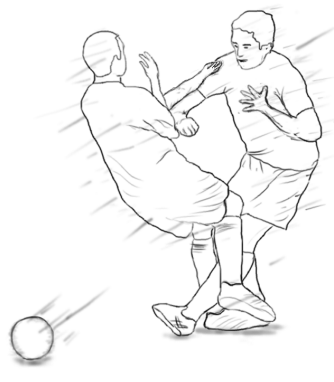

c)

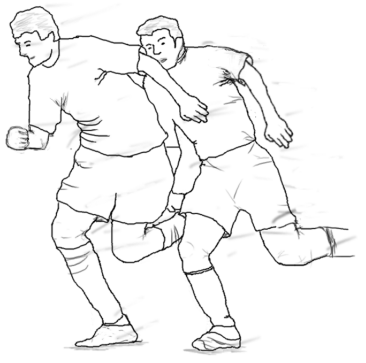

Fig. 3. Different situations of limb impacts: a) A high foot hits the opponent's chest instead of the ball (L3). b) Two players collide and hit each other's knee (L7). c) A player pushes his elbow into a chasing opponent's face (L4).

surface to accelerate the ball fast enough and therefore use a thinner coating for the head. Possibly non-breakable materials such as rubber, polyurethane or silicone are the ones of choice. Further aspects concerning weight and height are discussed in Sec. IV-C.

\section{Limb Impacts (L)}

Dangerous impacts caused by limbs, i.e. colliding legs or arms with the opponent's body can be roughly divided into

- Elbow checks (intended or unintended) to the other's face (Fig. 3c; L4, L10)

- A player sliding into or stepping on another player who is on the ground (Fig.4a; L5, L6)

- A leg hitting the opponent's trunk (Fig. 3a; L3)

- Legs or feet of two players colliding (Fig. 3b; L1, L2, L7, L8, L9)

The first class of impacts can be reduced to subhuman injury level by padding the robot's elbow. The other ones are caused by the boot which is the same for robots and humans. The enormous velocity of the kicking foot (see Sec. IV) can be fatal, so the robot must detect the absence of the human head absolutely reliably.

Impacts with other parts of the goalkeeper than the head are not sharply separable from the third injury source, where passive compliance in the joints is crucial to decouple the impact area from the rest of the robot. This protects both, the human and the robot from being injured/damaged (see Sec. IV-A). In other words, passive joint compliance is enhancing safety for both, the human and the robot. Obviously, this mechanism is limited as well: in an outstretched singular configuration, joint compliance has no effect and the Cartesian reflected inertia is vastly increasing. As for humans, this 
a)

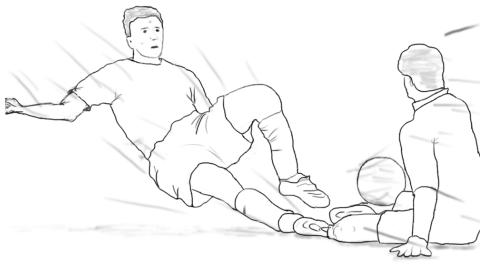

b)

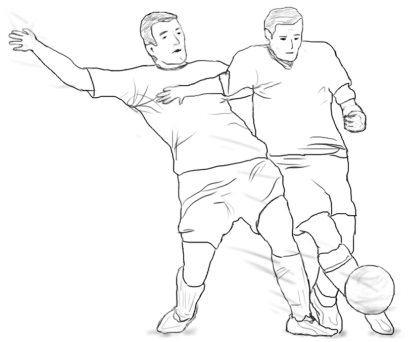

Fig. 4. a) A striker tries to reach the ball and slides into the sitting goalkeeper (L5). b) A typical situation with two players pushing each other while chasing the ball (P2).

configuration has to be avoided during such an impact under any circumstances to prevent both parties from damage.

\section{Being Hit by the Ball}

Being hit by a fast soccer ball can be a very painful experience. In order to analyze such an impact we carried out a one-dimensional simulation. The human head is modeled as a simple mass and the ball as a mass-spring system ${ }^{4}$, justified by high-speed camera recordings (Fig. 5a). Injury Severity is expressed by the so-called Head Injury Criterion (HIC), following the extended Prasad/Mertz curves ${ }^{5}$ for the conversion to probability of injury. This criterion is the most important severity index used in automobile crash-testing and was introduced to robotics in [19], [18]. In Fig. 5b, the resulting Head Injury Criterion is plotted against impact velocity and the probability of serious ${ }^{6}$ injury for different impact velocities is indicated. It shows that kicks, carried out by humans do not pose a serious threat, whereas increasing ball speed by only $50 \%$ would be already much more dangerous. These observations strictly forbid an approach to counterbalance lack of robot intelligence by simple power, i.e. no "brute force" is possible in robot-soccer. In addition to the potential threat posed to human heads by faster impacts, the joints of the robot can suffer damage from such fast kicks. This type of loading is mostly the same as if the robot kicks the ball and is discussed in Sec. IV.

\section{E. Secondary Impacts}

A situation more unlikely to happen but still worth to be mentioned are secondary impacts such as the ones dur-

\footnotetext{
${ }^{4}$ Because no adequate damping models are available, we neglect this effect.

${ }^{5}$ There exist various mappings to injury probability and interpretations of the HIC leading to different numerical values. However, we use one of them to show its extreme velocity dependency.

${ }^{6}$ An internationally established classification of injury severity is defined by the Abbreviated Injury Scale (AIS) [27]. Serious injury is indicated by $\mathrm{AIS}=3$.
}

a)
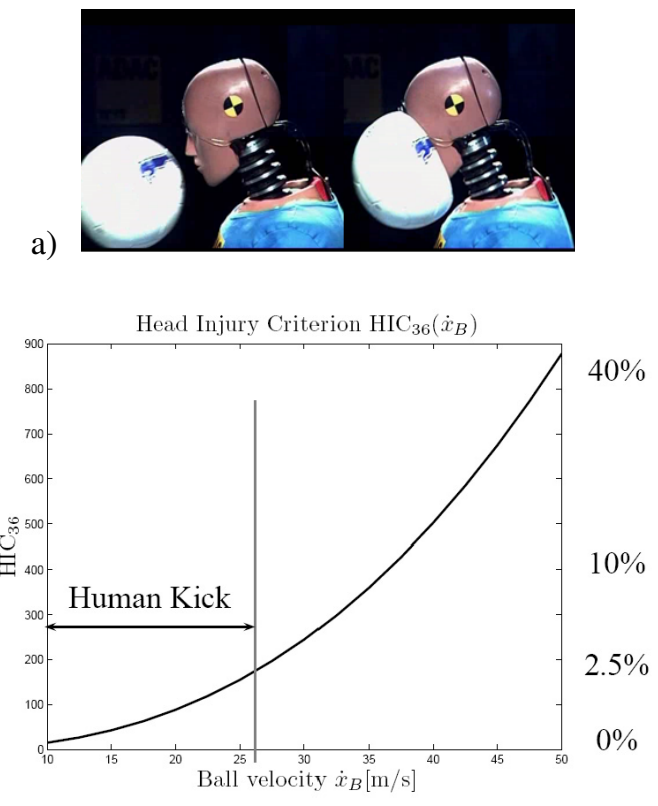

Fig. 5. a) Hitting a HybridIII dummy with a soccer ball. The impact is almost fully defined by the properties of the ball. The elasticity of the head can be neglected. Courtesy of the German Automobile Club (ADAC). b) The HIC as a function of impact velocity and resulting probability of serious $(\mathrm{AIS}=3$ ) injury.

ing heading duels, whereafter one of the players unluckily clashes against the goalpost. Additionally, a player could be pushed against the boards next to the field ${ }^{7}$. These secondary impacts are potentially dangerous to both, human and robot, so the robot should have sufficient understanding to avoid such situations if possible. In order to protect itself from being damaged, padding and compliant joints seem to be an adequate countermeasure.

\section{HOW BIG IS THE GAP?}

In order to illustrate what seems feasible with current technology by now, we simulated the DLR light-weight robot III (DLR-LWRIII) following a simplified planar trajectory (Fig. 6) to instep-kick a soccer ball. The inertial properties come quite close to the average human leg [28] $\left(\frac{L W R I I I}{L e g} \approx\right.$ $1.2)$ and therefore we can use it as a reasonable state-of-theart model for comparison. According to [2] the velocity of the ball can be expressed accurately enough by

$$
\dot{x}_{B}=\dot{x}_{F} \frac{m_{F}(1+e)}{m_{F}+m_{B}},
$$

where $\dot{x}_{B}$ and $\dot{x}_{F}$ are the velocities of the ball and foot, $m_{F}$ and $m_{B}=0.45 \mathrm{~kg}$ their effective masses and $e=0.5$ the coefficient of restitution ${ }^{8}$. Since the DLR-LWRIII has in outstretched position a reflected inertia of $\approx 4 \mathrm{~kg}$ in the impact direction, the velocity of the robot's end needs to be $\approx 0.75 \dot{x}_{B}$, leading with $16 \mathrm{~m} / \mathrm{s} \leq \dot{x}_{B} \leq 27 \mathrm{~m} / \mathrm{s}$ to

$$
12 \mathrm{~m} / \mathrm{s} \leq \dot{x}_{R} \leq 20.25 \mathrm{~m} / \mathrm{s} \text {. }
$$

\footnotetext{
${ }^{7}$ In new soccer arenas, tracks are usually left out so that this is definitely not too unlikely.

${ }^{8}$ The coefficient of restitution (COR) is the relation of the velocity before and after an impact.
} 


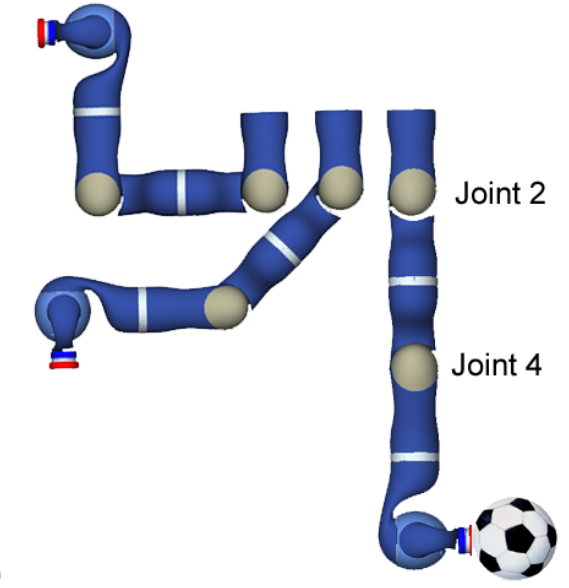

a)
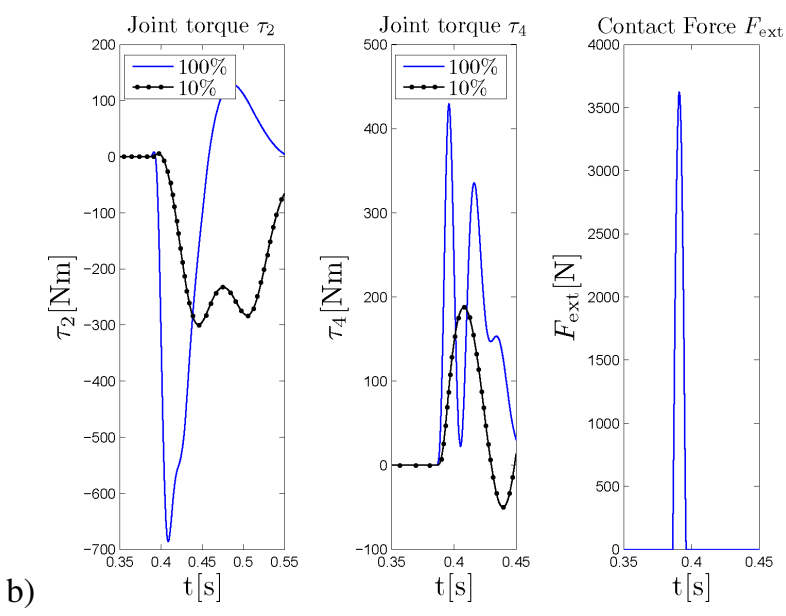

Fig. 6. a) Planar kick trajectory using joints 2 and 4 of the DLR-LWRIII. b) Joint torques of the DLR-LWRIII and contact force for $100 \%$ and $10 \%$ of the intrinsic joint stiffness caused by kicking a ball fast enough $\left(700^{\circ} / \mathrm{s}\right.$ joint velocity) to reach a ball velocity of $\dot{x}_{B}=27 \mathrm{~m} / \mathrm{s}$. The real maximum joint torque of the robot in the $2 \mathrm{nd}$ axis is $180 \mathrm{Nm}$ and in the 4 th axis $80 \mathrm{Nm}$.

According to [29], the stiffness of the soccer ball ranges from $17.7-43.7 \mathrm{kN} / \mathrm{m}$. A simple mass-spring model may be used to calculate a robotic kick. First, the ball will be hit in outstretched configuration with the maximum possible velocity of the real system to point out what could be done with a state-of-the-art arm designed for physical human-robot interaction. Then, the velocity of the robot will be upscaled to a sufficient value to hit the ball fast enough to accelerate the ball to the desired and necessary velocity common in soccer. Humans kick at joint velocities up to $24 \mathrm{rad} / \mathrm{s}$ for knee extension and with joint torques up to $280 \mathrm{Nm}$ [4]. Kicking a soccer ball at the maximum nominal joint velocity $\left(130^{\circ} / \mathrm{s}\right)$ of the DLR-LWRIII leads to a ball velocity of $\approx 4.5 \mathrm{~m} / \mathrm{s}$, i.e. six times slower than required and the joint torques are already reaching critical values ( $80 \%$ of maximum nominal torque).

\section{A. Actuators}

The previous result already shows that due to limitations in joint velocity of currently available robotic systems it would be impossible to accelerate the ball to its required velocity. For the real DLR-LWRIII the shown trajectory is feasible at maximum Cartesian velocity of $\approx 2.7 \mathrm{~m} / \mathrm{s}$, i.e. $4.5-7.5$ times too slow. Actually, humans kick at even faster Cartesian velocities because their reflected inertia is lower. It seems problematic to reach such velocities with classical serial motor/drive units without vastly enlarging the motor. This increase in robot mass naturally poses a greater potential threat to the human. Theoretically, one could realize a speedup for the DLR-LWRIII of two times the current maximum speed just out of the used gears but still, this is far too slow to come close to necessary velocities.

Hitting the ball at sufficient velocities yields joint torques and an external force as shown in Fig. 6 (right). This leads to a very problematic observation concerning joint torques caused by the impact. The lever of the external contact force is extremely high in outstretched configuration and results in naturally very high joint torques, potentially causing mechanical damage to the gear box and torque sensors by overload. This is confirmed by observations we made during robotdummy impacts presented in [14], where already at impact velocities of $\approx 1 \mathrm{~m} / \mathrm{s}$ exceedance of maximum nominal joint torques were observed. Furthermore, it was pointed out that via control (torque or impedance control) one is not able to react ${ }^{9}$ fast enough to prevent the robot from being damaged during faster and rigid impacts. Introducing passive variable compliant joints and decreasing the coupling of motor from link side inertia by adjusting very low stiffness seems crucial. Because of the intrinsically lower stiffness, peak joint torques would be diminished (Fig. 6b), preventing the robot from damage. However, the impact force $F_{\text {ext }}$ remains the same for both joint stiffnesses because during the very short duration of impact only the link side inertia counts and the motor is already decoupled by the intrinisc joint spring. Secondly, the manipulator would presumably be able to accelerate to the required velocity for such an instep kick. This is because the joint springs can store potential energy, which probably can be used to provide further torque while striking out for accelerating the link, if the motor reaches its maximum velocity.

\section{B. Soccer Kick with an Industrial Robot}

In order to show by a very intuitive experiment the performance limits of classical actuation, a soccer ball was kicked with a KUKA KR500 ${ }^{10}$ at maximum joint velocity, resulting in a Cartesian impact velocity of $3.7 \mathrm{~m} / \mathrm{s}$ (see Fig. 7 and especially the corresponding video attachment). The ball hits the ground after a flight of only $\approx 2 \mathrm{~m}$. In comparison, a human performed a kick as well and one can clearly see how slow and careful he hits the ball in order not to shoot farther. Additionally, a rather hard shot was taken to show the dramatic contrast to the robot. This example clearly gives a better feeling which limitations are still present and have to be overcome in current robotics to realize the desired properties.

\footnotetext{
${ }^{9}$ Even ideally fast detection cannot significantly diminish this deficit.

${ }^{10}$ This robot has a nominal payload of $500 \mathrm{~kg}$.
} 


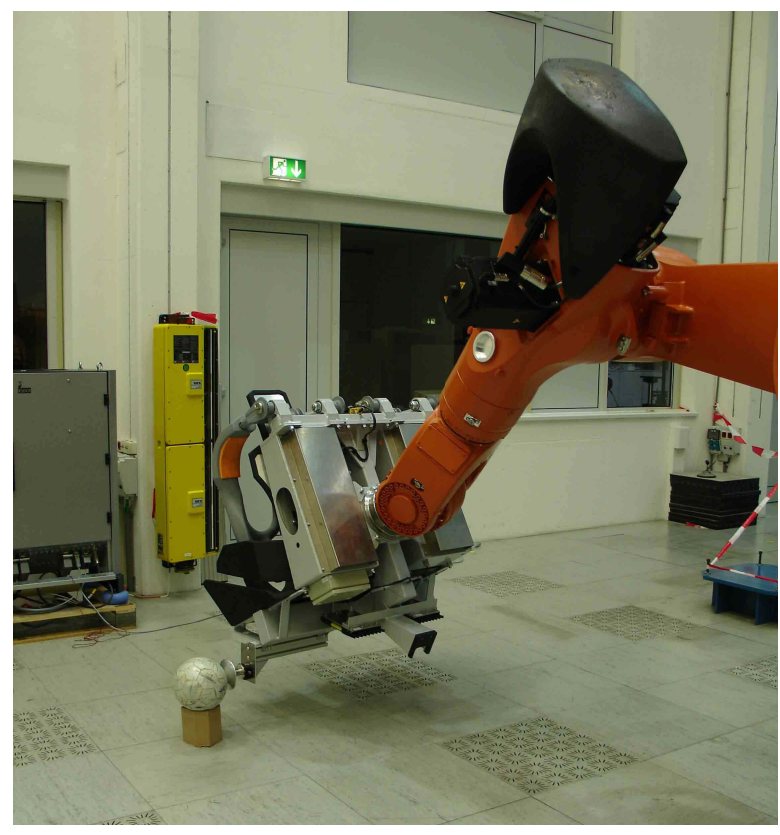

Fig. 7. A football kick with a KUKA KR500.

\section{Further Aspects}

Besides the heavy interactions described in Sec. III, of which most ones are fouls or tackles, several other comparably light contacts occur in soccer. In almost all matches, situations, in which two players parallely run to a ball and mutually obstruct each other could be observed (Fig. 4b; P1, P2, P6). Light pushes (P4, P5) without any consequences happen as well as entangling arms in crowded situations (P3). This raises the question whether a soccer robot would benefit from a touch sensitive skin.

Another aspect not fully discussed in this paper is the possible necessity of specialized team role robots. Because of the varying loading of players in different positions, having different types of players is beneficial. Especially goalkeeper sprint seldom but often dive and fall on the whole body when fending a ball, whereas field players are posed to sustained loaded and duels. According to [20] injury severity and mechanisms highly depend on the position of the player, pointing out that goalkeeper have been shown to have more head, face, neck, and upper extremity injuries than lower extremity injuries. Another very intuitive reason to design different player types is that because of their inertial properties, massive and hence slow players cannot fulfill the role of a fast and flexible game maker. It can often be observed in real world soccer that manipulability of the body is more important than simple speed and strength. According to [30] the average height of players is differentiated between striker $(\approx 176 \mathrm{~cm})$, defender $(\approx 185 \mathrm{~cm})$ and goalkeeper $(\approx 190 \mathrm{~cm})$, clearly indicating the necessary specialization for each position. An obvious reason for this difference in height are headings or for a goal keeper reaching the kick by length [30]. Furthermore, there is the natural advantage of heterogeneity and diversity within the team.

\section{CONCLUSion}

We located and analyzed safety critical scenes of soccer, exemplified by two matches of the FIFA World Cup 2006 in Germany. A typical instep soccer kick carried out by a state-of-the-art robot was simulated to point out some critical constraints of current robotic systems. This led to the necessity of new actuation paradigms, meaning introduction of passive and adjustable (variable) compliance into the joints. This feature is crucial to ensure both, safety to the human and robot soccer player. Furthermore, it could contribute to accelerate the soccer ball to the desired range of speed. Safety issues are already treated in current robotic research and the basic injury mechanisms are under investigation. This seems to be a good foundation also for robotic soccer players but still, it appears that major technological novelties are required. Even though it is highly speculative one could imagine that classical electromechanical actuation turns out to be principally insufficient with respect to desired requirements. Therefore, it might be useful to think about other concepts like biomechanical actuation.

\section{ACKNOWLEDGMENT}

We would especially like to thank Christian Ott and Alin Albu-Schäffer for the fruitful discussions, leading to very interesting formulations of problems and deeper insight.

Special thanks also go to Christoph Brachmann for sketching the soccer illustrations in this paper.

\section{REFERENCES}

[1] H. Kitano and M. Asada, "RoboCup Humanoid Challenge: That's One Small Step for A Robot, One Giant Leap for Mankind," in International Conference on Intelligent Robots and Systems, Victoria, 1998, pp. 419-424.

[2] A. Lees and L. Nolan, "The Biomechanics of Soccer: A Review," Journal of Sport Sciences, 1998.

[3] Fédération Internationale de Football Association, "Laws of the Game 2006," 2006, http://www.fifa.com/documents/fifa/laws/LOTG2006_e.pdf.

[4] H. Nunome, T. Asai, and Y. I. S. Sakurai, "Three-dimensional kinetic analysis of side-foot and instep soccer kicks," Medicine \& Science in Sports \& Excercise, vol. 34, no. 12, pp. 2028-2036, 2002.

[5] G. Hirzinger, N. Sporer, A. Albu-Schäffer, R. Krenn, A. Pascucci, and M. Schedl, "DLR's Torque-Controlled Light Weight Robot III Are We Reaching The Technological Limits now?" ICRA2002, pp. 1710-1716, 2002.

[6] PHRIENDS, "PHRIENDS web site," 2007, http://www.phriends.eu/.

[7] SMErobot, "SMErobot web site," 2007, http://www.smerobot.org/.

[8] C. Ott, O. Eiberger, W. Friedl, B. Bäuml, U. Hillenbrand, C. Borst, A. Albu-Schäffer, B. Brunner, H. Hirschmüller, S. Kielhöfer, R. Konietschke, M. Suppa, T. Wimböck, F. Zacharias, and G. Hirzinger, "A Humanoid Two-Arm System for Dexterous Manipulation,” 2006.

[9] H. Kawasaki, T. Komatsu, and K. Uchiyama, "Dexterous anthropomorphic robot hand with distributed tactile sensor: Gifu hand II," IEEE/ASME Transactions Mechatronics, vol. 7, pp. 296-303, 2002.

[10] W. Townsend and J. Salisbury, "Mechanical Design for WholeArm Manipulation. Robots and Biological Systems: towards a new Bionics?" pp. 153-164, 1993.

[11] K. Kaneko, F. Kanehiro, S. Kajita, H. Hirukawa, T. Kawasaki, M. Hirata, K. Akachi, and T. Isozumi, "Humanoid Robot HRP-2," ICRA2004, pp. 1083-1090, 2004.

[12] N. Hogan, "Impedance Control: An Approach to Manipulation: Part I - Theory, Part II - Implementation, Part III - Applications," Journal of Dynamic Systems, Measurement and Control, vol. 107, pp. 1-24, 1985. 
[13] J. Craig and M. Raibert, "A Systematic Method for Hybrid Position/Force Control of a Manipulator," IEEE Computer Software Applications Conference, pp. 446-451, 1979.

[14] S. Haddadin, A. Albu-Schäffer, and G. Hirzinger, "Safety Evaluation of Physical Human-Robot Interaction via Crash-Testing," Robotics: Science and Systems Conference, 2007.

[15] G. Tonietti, R. Schiavi, and A. Bicchi, "Design and Control of a Variable Stiffness Actuator for Safe and Fast Physical Human/Robot Interaction," Proc. ICRA 2005, pp. 528-533, 2005.

[16] B. Vanderborght, B. Verrelst, R. Van Ham, M. Van Damme, D. Lefeber, B. Meira Y Duran, and P. Beyl, "Exploiting Natural Dynamics to Reduce Energy Consumption by Controlling the Compliance of Soft Actuators," The International Journal of Robotics Research, vol. 25, pp. 343-358, 2006.

[17] I. Asimov, The Caves Of Steel, A Robot Novel, 1954.

[18] A. Bicchi and G. Tonietti, "Fast and Soft Arm Tactics: Dealing with the Safety-Performance Trade-Off in Robot Arms Design and Control," IEEE Robotics and Automation Magazine, vol. 11, pp. 22-33, 2004.

[19] M. Zinn, O. Khatib, and B. Roth, "A New Actuation Approach for Human Friendly Robot Design," The International Journal of Robotics Research, vol. 23, pp. 379-398, 2004.

[20] A. McGrath and J. Ozanne-Smith, "Heading Injuries out of Soccer: A Review of the Literature," vol. 125, 1997.

[21] K. Knobloch, D. Rossner, M. Jagodzinski, J. Zeichen, T. Gössling, S. Martin-Schmitt, M. Richter, and C. Krettek, "Prävention von Schulsportverletungen - Analyse von Ballsportarten bei 2234 Verletzungen," Sportverletzungen Sportschäden, vol. 19, pp. 82-88, 2005.

[22] J. Newman and N. Shewchenko, "A Proposed New Biomechanical Head Injury Assessment Function - The Maximum Power Index," SAE paper 2000-01-SC16 Stapp Car Crash Journal, vol. 44, pp. 215-247, 2000.

[23] N. Shewchenko, N. Withnall, M. Keown, R. Gittens, and J. Dvorak, "Heading in Football. Part 1: Development of Biomechanical Methods to investigate Head Response," British Journal Sports Medicine, vol. 39, pp. 10-25, 2005.

[24] H.-D. Burkhard, D. Duhaut, M. Fujita, P. Lima, R. Murphy, and R. Rojas, "The Road to RoboCup 2050," IEEE Robotics and Automation Magazine, vol. 9(2), pp. 31-38, 2002.

[25] R. Durašković, A. Joksimović, and S. Joksimović, "Weight-Height Parameters of the 2002 World Football Championship Participants," Physical Education and Sport, vol. 2, pp. 13-24, 2004.

[26] J. Melvin, "Human Tolerance to Impact Conditions as related to Motor Vehicle Design," SAE J885 APR80, 1980.

[27] American Association for Automotive Medicine, "The Abbreviated Injury Scale," 1980.

[28] R. Chandler, C. Clauser, J. McConville, H. Reynolds, and J. Young, "Investigation of Inertial Properties of the Human Body," Aerospace Medical Research Laboratory, Tech. Rep., 1975.

[29] N. Shewchenko, N. Withnall, M. Keown, R. Gittens, and J. Dvorak, "Heading in Football. Part 3: Effect of Ball Properties," British Journal Sports Medicine, vol. 39, pp. 33-39, 2005.

[30] J. Wesson, The Science of Soccer. Bristol: IOP Publishing Ltd., Dirac House, Temple Bacl, 2002. 